\title{
On the non-uniqueness of local quasi-geoids computed from terrestrial gravity anomalies
}

\author{
I. Prutkin · R. Klees
}

Received: 16 October 2006 / Accepted: 12 April 2007 / Published online: 8 May 2007

(C) Springer-Verlag 2007

\begin{abstract}
We consider the problem of local (quasi-)geoid modelling from terrestrial gravity anomalies. Whereas this problem is uniquely solvable (up to spherical harmonic degree one) if gravity anomalies are globally available, the problem is non-unique if gravity anomalies are only available within a local area, which is the typical situation in local/regional gravity field modelling. We derive a mathematical description of the kernel of the gravity anomaly operator. The non-uniqueness can be removed using external height anomaly information, e.g., provided by GPS-levelling. The corresponding problem is formulated as a Cauchy problem for the Laplace equation. The existence and uniqueness of the solution of the Cauchy problem is guaranteed by the Cauchy-Kowalevskaya theorem. We propose several numerical procedures to compute the solution of the Cauchy problem from given differences between gravimetric and geometric height anomalies. We apply the numerical techniques to real data over the Netherlands and Germany. We show that we can compute a unique quasi-geoid from observed gravimetric and geometric height anomalies, which agree with the data within the expected noise level. We conclude that observed differences between gravimetric height anomalies and geometric height anomalies derived from GPS and levelling cannot only be attributed to systematic errors in the data sets, but are also caused by the intrinsic non-uniqueness of the problem of local quasi-geoid modelling from gravity anomalies. Hence, GPS-levelling data are necessary to get a unique solution, which also implies that they should not be
\end{abstract}

I. Prutkin $\cdot$ R. Klees $(\varangle)$

Delft Institute of Earth Observation and Space Systems (DEOS), Delft University of Technology, Kluyverweg 1,

2629 HS Delft, The Netherlands

e-mail: r.klees@tudelft.nl used to validate local quasi-geoid solutions computed on the basis of gravity anomalies.

Keywords Local gravity field modelling .

Non-uniqueness · Cauchy problem for the Laplace

equation · GPS-levelling data $\cdot$ Quasi-geoid

\section{Introduction}

The combination of gravimetrically derived quasi-geoid heights over a target area with ellipsoidal heights provided by GPS has become a standard procedure in quasi-geoid and height reference surface modelling (e.g., Fotopoulos 2005 and many others). The main motivation for combining these types of data seems to be: (i) to improve the quality of the gravimetrically derived quasi-geoid model for the target area, or (ii) to facilitate the more direct transformation of GPS ellipsoidal heights into the heights of the adopted national height system (cf. Featherstone 1998).

The reference surface for a national height system is the quasi-geoid for normal and normal-orthometric heights or the geoid for orthometric heights; in practice, there may be significant differences between the quasi-geoid/geoid and the national height reference surface. For instance, differences may be caused by subsidence/uplift of the markers of the first-order height control network (cf. Lysaker et al. 2007), or by simplifications used in the computation of geopotential numbers (e.g., Vanicek et al. 1980).

In the following, we assume: (i) the national height system uses normal heights and (ii) from gravity anomalies and geopotential numbers, a global gravity field model, and a digital terrain model, a quasi-geoid has been computed for the target area. From levelling and gravimetry along the levelling lines, 
normal heights with respect to the datum point of the national height system can be determined. When subtracted from GPS ellipsoidal heights, we obtain what here we call geometric height anomalies. From terrestrial (or airborne) gravity anomalies, a global gravity field model, and a digital terrain model, we obtain so-called gravimetric height anomalies following one of the many procedures of quasi-geoid determination; we will not debate the different approaches here.

When geometric height anomalies are compared with gravimetric height anomalies, one often observes systematic differences, which can be quite large, on the order of several decimeters (e.g., de Min 1996; Featherstone 1998; Fotopoulos et al. 2003). They are commonly attributed to datum inconsistencies, different tidal systems, long-wavelength geoid errors, distortions in the vertical network, and GPS errors. Therefore, the model

$\zeta_{\text {grav }}-\zeta_{\text {geom }}=0$,

where $\zeta_{\text {geom }}$ is the geometric height anomaly and $\zeta_{\text {grav }}$ is the gravimetric height anomaly, is commonly augmented by a so-called "corrector surface" term $f$ to account for the systematic differences between gravimetric and geometric height anomalies, i.e.,

$\zeta_{\text {grav }}-\zeta_{\text {geom }}-f=0$.

Once a so-called "corrector surface" is available, the transformation of a measured GPS ellipsoidal height $h$ at a new point $P$, into the national height $H$ is obtained according to

$H_{P}=h_{P}-\zeta_{\text {grav }, P}-f_{P}$.

Usually, Eq. (2) is used as functional model, and the parameters of the "corrector surface" can be estimated by leastsquares. A more general model, involving a stochastic signal term, has been introduced by Kotsakes and Sideris (1999), see also Fotopoulos (2005) and Grebenitcharsky et al. (2005). An alternative approach to the combination of geometric and gravimetric height anomalies with the goal to obtain an optimal transformation of GPS ellipsoidal heights into the national height system, has been introduced by Dinter et al. (1997) and refined by Jäger $(1999,2000)$ and Jäger and Schneid (2001). Least squares collocation (LSC) is also commonly used, but numerous other approaches have been trialled (e.g., Soltanpour et al. 2006; Lysaker et al. 2007).

In practice, the "corrector surface" $f$ is parameterized in different ways. For instance, de Min (1996) uses a bivariate linear algebraic polynomial in latitude and longitude for the area of the Netherlands; de Bruijne et al. (1997) use a linear combination of a bilinear polynomial and a trigonometric polynomial in latitude and longitude with 28 parameters for the North Sea region; Grebenitcharsky et al. (2005) use a four-parameter trigonometric model for Canada originally suggested by Heiskanen and Moritz (1967); Jäger (1999) uses a bivariate algebraic polynomial defined on triangular meshes for parts of Germany; Featherstone (2000) compares splines under tension and LSC; Nahavandchi and Soltanpour (2006) use, among others, cubic splines. The proper choice of a corrector surface, which is a model identification problem, is still an open issue.

There is, however, another contributor to the systematic differences between gravimetric and geometric height anomalies, which - as far as we know-has not been addressed yet in the literature. It is caused by a non-trivial kernel of the operator that maps height anomalies within a local area (or, equivalently, disturbing potentials) into gravity anomalies.

The mathematical foundation of gravity field determination from gravity anomalies and geopotential numbers is the scalar Molodensky problem (e.g., Sacerdote and Sanso 1987). We know that if global data are available, this problem has a non-trivial null space, which means that not all parameters of the Earth's gravity field can be uniquely determined from the given data. However, the non-uniqueness is only related to the terms of degree-one of the spherical harmonic expansion of the gravity field. Over a local area, this non-uniqueness appears as an almost constant bias in the computed quasi-geoid, which can easily be parameterized using a low-degree polynomial.

However, the situation changes if data is only available over a limited area of the Earth's surface, which is typical for local gravity field modelling. Then, the null space is much larger, comprising all non-zero harmonic functions that produce zero gravity anomalies over the local area. Geometric height anomalies are unique. Therefore, differences between geometric and gravimetric height anomalies also contain the part of the gravity field that cannot be determined from terrestrial gravity data. In that context, it is the role of geometric height anomalies to determine the part of the disturbing potential that cannot be determined from terrestrial gravity anomalies.

In this paper, we will construct a non-trivial function, which is harmonic in the neighborhood of the target area and has an almost zero gravity anomaly signal over the target area. We compute this function using differences between gravimetric and geometric height anomalies as data. Different areas are investigated, including the Netherlands and Germany. We show that the function represents the differences between geometric and gravimetric height anomalies, with residuals on the order of the data noise.

The paper is organized as follows: in Sect. 2, we give a description of the kernel of the gravity anomaly operator. In particular, we show that there exists a unique non-trivial harmonic function in the neighborhood of the target area, which produces almost zero gravity anomalies. This function is the solution of a Cauchy problem for the Laplace equation and can be determined from GPS-levelling data.

In Sect. 3, we propose a simple numerical procedure to obtain an approximate solution of the Cauchy problem for 
the Laplace equation in the neighborhood of the target area from observed differences between geometric and gravimetric height anomalies. This simple scheme can be applied to relatively small areas of several thousand square kilometers. More general numerical procedures, which can be applied to local areas of arbitrary size, are the subject of Sect. 4.

The numerical procedures of Sects. 3 and 4 are then applied to real data over three test areas in Sect. 5. The fit of the function to the data depends on the adopted noise level. For the Netherlands, we compute a solution that fits the data with an RMS of $0.3 \mathrm{~cm}$. For Germany, the fit is $0.7 \mathrm{~cm}$. We also compute a solution for a rectangular area within Germany of $2^{\circ} \times 1.75^{\circ}$, following a simpler numerical procedure, which exploits the rectangular shape of the target area. The RMS fit with the data is $0.7 \mathrm{~cm}$. Section 6 contains a summary and the main conclusions.

\section{Approximate description of the kernel}

Suppose, $T_{1}$ is the (residual) disturbing potential, corresponding to gravity anomaly data, and $T_{2}$ is the (residual) disturbing potential, obtained from GPS-leveling data by means of Bruns's formula. With the term residual we mean that the contribution of a global gravity field model and a model of the topography are subtracted from the data, and, once a solution has been obtained, are restored (remove-restore procedure, see also Sjöberg 2005).

Let us assume that both solutions satisfy the given gravity data. Then, on the part $S$ of the telluroid surface that corresponds to the local area, it is (in spherical approximation)

$\Delta g=-\left.\frac{\partial T_{1}}{\partial r}\right|_{S}-\left.\frac{2}{r} T_{1}\right|_{S}=-\left.\frac{\partial T_{2}}{\partial r}\right|_{S}-\left.\frac{2}{r} T_{2}\right|_{S}$.

According to Eq. (4), the difference $\tilde{T}=T_{1}-T_{2}$ corresponds to zero gravity anomaly data, i.e., it belongs to the kernel of the operator $D_{\Delta g}$ that maps the disturbing potential into gravity anomalies according to $\Delta g=D_{\Delta g} T$. From Eq. (4), we obtain:

$\left.\frac{\partial\left(T_{1}-T_{2}\right)}{\partial r}\right|_{S}=\left.\frac{2}{r}\left(T_{2}-T_{1}\right)\right|_{S}$

The differences between (residual) gravimetric height anomalies $\zeta_{1}$ and (residual) geometric height anomalies $\zeta_{2}$ are usually below $1 \mathrm{~m}$. Then, the right-hand side of Eq. (5) is of the order of $0.3 \mathrm{mGal}$. This is below the level of accuracy of gravity anomaly data usually used in local gravity field modelling. Therefore, Eq. (5) can be approximated by

$\left.\frac{\partial\left(T_{1}-T_{2}\right)}{\partial r}\right|_{S}=\left.\frac{\partial \tilde{T}}{\partial r}\right|_{S}=0$.

The function $\tilde{T}$ should also be harmonic outside the telluroid. Suppose its restriction to the local telluroid area $S$ is equal to $f$. Then, we obtain the following system of boundary conditions for the harmonic function $\tilde{T}$ :

$\left.\frac{\partial \tilde{T}}{\partial r}\right|_{S}=0,\left.\quad \tilde{T}\right|_{S}=f$.

Since $\tilde{T}$ is harmonic, we end up with the following problem to be solved:

$$
\begin{aligned}
\Delta \tilde{T} & =0, \quad \text { in } D, \\
\left.\frac{\partial \tilde{T}}{\partial r}\right|_{S} & =0, \\
\left.\tilde{T}\right|_{S} & =f,
\end{aligned}
$$

where $D \subset \mathbb{R}^{3}$ is a suitably chosen vicinity of the local area $S$. Equation (8) is a Cauchy problem for the Laplace equation (Jost 2002). The existence and uniqueness of a solution in the vicinity of the local area $S$ is guaranteed by the Cauchy-Kovalevskaya theorem (e.g., Ebenfelt and Shapiro 1995). The function $f$ is the difference between the gravimetric and geometric height anomalies scaled by the normal gravity at the telluroid. In practice, $f$ is given at a set of points distributed over the target area $S$. The numerical solution of Eq. (8) is the subject of Sect. 4.

For many practical applications, we can replace the problem in Eq. (8) by a simpler problem. To obtain its mathematical formulation, we consider the Taylor expansion of $\tilde{T}$ in the radial direction:

$\tilde{T}=\left.\tilde{T}\right|_{S}+\left.\frac{\partial \tilde{T}}{\partial r}\right|_{S} \cdot \delta r+\mathcal{O}\left(\delta r^{2}\right)$,

where $\delta r$ is the increment in the radial direction. The second term on the right-hand side of Eq. (9) is equal to zero, according to Eq. (7). If we omit quadratic and higher order terms, we obtain a function $\hat{T}$, which approximates every function from the kernel of the operator $D_{\Delta g}$ with accuracy $\mathcal{O}\left(\delta r^{2}\right)$, and satisfies the stronger condition

$$
\frac{\partial \hat{T}}{\partial r} \equiv 0 .
$$

Such a function can be easily described: it does not depend on the radial distance $r$. If we write the expression of the Laplace operator in spherical coordinates $(r, \theta, \lambda)$ :

$$
\begin{aligned}
\Delta U= & \frac{1}{r^{2}} \frac{\partial}{\partial r}\left(r^{2} \frac{\partial U}{\partial r}\right)+\frac{1}{r^{2} \sin \vartheta} \frac{\partial}{\partial \vartheta}\left(\sin \vartheta \frac{\partial U}{\partial \vartheta}\right) \\
& +\frac{1}{r^{2} \sin ^{2} \vartheta} \frac{\partial^{2} U}{\partial \lambda^{2}},
\end{aligned}
$$

then, to be harmonic, the function $\hat{T}$ satisfies the differential equation

$$
\frac{1}{\sin \vartheta} \frac{\partial}{\partial \vartheta}\left(\sin \vartheta \frac{\partial \hat{T}}{\partial \vartheta}\right)+\frac{1}{\sin ^{2} \vartheta} \frac{\partial^{2} \hat{T}}{\partial \lambda^{2}}=0
$$


in the area $S$. Moreover, the function $\hat{T}$ takes boundary values:

$\left.\hat{T}\right|_{\partial S}=\left.f\right|_{\partial S}$,

where $\partial S$ is the boundary of the target area $S$.

The solution $\hat{T}$ of Eqs. (11) and (12) provides an approximation of the solution $\tilde{T}$, Eq. (8). $\hat{T}$ may be used if the size of the local area $S$ is not too large, e.g., $2^{\circ} \times 2^{\circ}$. The numerical solution of Eqs. (11) and (12) is the subject of Sect. 3.

\section{Computation of the function $\hat{T}$}

In this section, we study three different approaches to find a solution of the problem

$$
\begin{aligned}
& \frac{1}{\sin \vartheta} \frac{\partial}{\partial \vartheta}\left(\sin \vartheta \frac{\partial \hat{T}}{\partial \vartheta}\right)+\frac{1}{\sin ^{2} \vartheta} \frac{\partial^{2} \hat{T}}{\partial \lambda^{2}}=0, \text { in } S, \\
& \left.\hat{T}\right|_{\partial S}=\left.f\right|_{\partial S .}
\end{aligned}
$$

(i) the method of finite differences, (ii) the method of finite elements, and (iii) the piecewise finite difference method. To keep the numerics simple, we consider the area $S$ to be a part of the surface of a sphere with radius $R$, denoted $\sigma_{R}$.

Assume that $S$ is a rectangular area on the sphere, i.e., bounded by parallels and meridians. Then, the most natural and simple approach to solve Eqs. (11) and (12) are finite difference methods. The boundary data $\left.f\right|_{\partial S}$ could be obtained from the data $f$ on $S$ by some form of interpolation. For irregular boundaries $\partial S$, finite differences are not the best choice, so finite element methods (FEM) are preferred. Many commercial and free FEM software packages are available to perform the computations, e.g., NASTRAN, ABAQUS, MODULEF, SLEFEA, and FreeFEM.

For those who do not want to make use of these packages, there is an alternative, which we call the piecewise finite difference method. Suppose that data are given on the area $S$, and $S \subseteq S_{0}, S_{0}=\cup_{i} S_{i}$, where every $S_{i}$ represents a rectangle on the sphere bounded by parallels and meridians. Then we could solve Eqs. (11) and (12) for each rectangle. To do this, we interpolate the observed data $f$ to the boundary $\partial S_{i}$ of each rectangle $S_{i}$ (e.g., using thin-plate splines), and compute a solution for this sub-area using finite differences.

In this way, we obtain a solution for each rectangle $S_{i}$ that satisfies Eq. (11) inside the rectangle. The total solution is, however, only continuous in $S$. To refine the solution, we find all interior points of $S_{0}$, take the piecewise solutions as initial approximations, and apply an iterative procedure to solve Eqs. (11) and (12) with finite differences for interior points.

\section{Computation of the function $\tilde{T}$}

Equation (13) is an approximation of the more general Cauchy problem, Eq. (8), which may be sufficiently accurate provided that the local area $S$ is not too large (e.g., not larger than $2^{\circ} \times 2^{\circ}$ ). For larger areas, we have to solve Eq. (8). As in Sect. 3, we assume that the Cauchy data are given on a sphere, i.e., the local area $S$ is considered to be a sphere with radius $R, \sigma_{R}$.

The proposed numerical techniques to solve Eq. (8) are two-step procedures. The first step is common to all of them. Its goal is to obtain an initial solution, which is refined in the second step. The initial solution is obtained by applying the FEM to Eq. (13). This provides a first-order approximation $\hat{T}$ to $\tilde{T}$. The differences $\delta f:=f-\left.\hat{T}\right|_{S}$ will be very small, say, of the order of $10-20 \mathrm{~cm}$. Moreover, $\delta f$ will be zero on the boundary $\partial S$.

We set $\tilde{T}=\hat{T}+u$ and determine $u$ as solution of the problem

$$
\begin{aligned}
\Delta u & =0, \quad \text { in } D, \\
\left.\frac{\partial u}{\partial r}\right|_{S} & =0, \\
\left.u\right|_{S} & =\delta f,
\end{aligned}
$$

which is the second step of the procedure. Once Eq. (14) has been solved, we obtain the solution of Eq. (8) from $\tilde{T}=$ $\hat{T}+u$.

Solving the Cauchy problem, Eq. (14), may be a tedious task, as the problem is known to be extremely ill-conditioned (Hadamard 1921). Therefore, we do not solve this problem directly, but first solve the problem

$\Delta u=0, \quad$ in $D$,

$\left.u\right|_{S}=\delta f$,

and observe the boundary condition

$\left.\frac{\partial u}{\partial r}\right|_{S}=0$

using the idea of reflexion, or in the spherical case, the Kelvin transformation (e.g., Ito 1980).

We propose three approaches to compute the function $u$ : (i) the single-layer approach, (ii) the modified single-layer approach, and (iii) the Poisson integral approach.

\subsection{Single-layer approach}

The solution of Eq. (14) is represented as the sum of two single-layer potentials with unknown surface densities $\mu_{1}$ and $\mu_{2}$, defined on the spheres $\sigma_{R_{1}}$ and $\sigma_{R_{2}}$ with radii 
$R_{1}=R+h$ and $R_{2}=R-h$, respectively, i.e.,

$u(x)=u_{1}(x)+u_{2}(x), \quad x \in D$,

$u_{1}(x)=\int_{\sigma_{R_{1}}} \frac{\mu_{1}(y)}{|x-y|} \mathrm{d} \sigma_{R_{1}}(y)$,

$u_{2}(x)=\int_{\sigma_{R_{2}}} \frac{\mu_{2}(y)}{|x-y|} \mathrm{d} \sigma_{R_{2}}(y)$.

The value $h$ is chosen appropriately, i.e., such that the spherical shell $R-h \leq r \leq R+h$ is contained in $D$. The functions $u_{1}$ and $u_{2}$ are harmonic inside the spherical shell. With

$u_{1}(x)=\sum_{n, m} \bar{c}_{n, m}^{\left(u_{1}\right)} \bar{Y}_{n, m}(\hat{x})\left(\frac{|x|}{R_{1}}\right)^{n}$,

$u_{2}(x)=\sum_{n, m} \bar{c}_{n, m}^{\left(u_{2}\right)} \bar{Y}_{n, m}(\hat{x})\left(\frac{R_{2}}{|x|}\right)^{n+1}$,

where $\hat{x}=\frac{x}{|x|}$ is a point on the unit sphere, and

$$
\begin{aligned}
& \mu_{1}(y)=\sum_{n, m} \bar{c}_{n, m}^{\left(\mu_{1}\right)} \bar{Y}_{n, m}(\hat{y}), \\
& \mu_{2}(y)=\sum_{n, m} \bar{c}_{n, m}^{\left(\mu_{2}\right)} \bar{Y}_{n, m}(\hat{y}),
\end{aligned}
$$

we obtain the following relation between the spherical harmonic coefficients of $u_{1}, \mu_{1}$ and $u_{2}, \mu_{2}$, respectively:

$\begin{aligned} \bar{c}_{n, m}^{\left(u_{1}\right)} & =\frac{4 \pi R_{1}}{2 n+1} \bar{c}_{n, m}^{\left(\mu_{1}\right)}, \\ \bar{c}_{n, m}^{\left(u_{2}\right)} & =\frac{4 \pi R_{2}}{2 n+1} \bar{c}_{n, m}^{\left(\mu_{2}\right)} .\end{aligned}$

The function $\delta f$, which takes zero values on $\partial S$, is continuously extended by zero to the whole surface of the sphere $\sigma_{R}$; this function is called $g$. Then, the boundary conditions in Eq. (14) read, in terms of spherical harmonic coefficients

$$
\begin{aligned}
& n\left(\frac{R}{R_{1}}\right)^{n-1} \bar{c}_{n, m}^{\left(\mu_{1}\right)}-(n+1)\left(\frac{R_{2}}{R}\right)^{n+2} \bar{c}_{n, m}^{\left(\mu_{2}\right)}=0 \\
& R_{1}\left(\frac{R}{R_{1}}\right)^{n} \bar{c}_{n, m}^{\left(\mu_{1}\right)}+R_{2}\left(\frac{R_{2}}{R}\right)^{n+1} \bar{c}_{n, m}^{\left(\mu_{2}\right)}=\frac{2 n+1}{4 \pi} \bar{c}_{n, m}^{(g)},
\end{aligned}
$$

where $\bar{c}_{n, m}^{(g)}$ are the spherical harmonic coefficients of the function $g$, defined as

$g(y)=\sum_{n=0}^{\infty} \sum_{m=-n}^{n} \bar{c}_{n, m}^{(g)} \bar{Y}_{n, m}(\hat{y}), \quad \hat{y}=\frac{y}{|y|}$.

From Eqs. (27) and (28), we obtain immediately an unique representation of the spherical harmonic coefficients of the single-layer densities

$\bar{c}_{n, m}^{\left(\mu_{1}\right)}=\bar{c}_{n, m}^{(g)} \frac{n+1}{4 \pi R}\left(\frac{R_{1}}{R}\right)^{n-1}$,
$\bar{c}_{n, m}^{\left(\mu_{2}\right)}=\bar{c}_{n, m}^{(g)} \frac{n}{4 \pi R_{2}}\left(\frac{R}{R_{2}}\right)^{n+1}$,

and, when observing Eqs. (25) and (26), of the potentials $u_{1}$ and $u_{2}$,

$\bar{c}_{n, m}^{\left(u_{1}\right)}=\bar{c}_{n, m}^{(g)} \frac{n+1}{2 n+1}\left(\frac{R_{1}}{R}\right)^{n}$,

$\bar{c}_{n, m}^{\left(u_{2}\right)}=\bar{c}_{n, m}^{(g)} \frac{n}{2 n+1}\left(\frac{R}{R_{2}}\right)^{n+1}$.

Equations (18), (31), and (32) provide the solution $u$ of problem Eq. (14):

$u(x)=\sum_{n, m} \bar{c}_{n, m}^{(g)} \frac{1}{2 n+1} \bar{Y}_{n, m}(\hat{x})\left((n+1)\left(\frac{|x|}{R}\right)^{n}+n\left(\frac{R}{|x|}\right)^{n+1}\right)$.

From Eq. (33) we also deduce that the solution exists and is unique. Note that by construction, $u$, Eq. (33), fulfils the boundary condition, Eq. (17).

From a numerical point of view, Eq. (33) is less efficient, as harmonic expansions up to very high degree may be necessary to represent the function $g$ with sufficient accuracy. For instance, in the Netherlands $\left(\approx 42,000 \mathrm{~km}^{2}\right)$, about 500 GPS-levelling points are currently available, i.e., about 1 point per $10 \mathrm{~km}$. To resolve this signal, a spherical harmonic expansion complete to degree 2000 would be necessary. Therefore, in Sects. 4.2 and 4.3, we propose an alternative to Eq. (33) that is numerically simpler.

\subsection{Modified single-layer approach}

An alternative to the approach proposed in Sect. 4.1 starts with Eq. (14). The function $u$ is represented as $u=\frac{u_{1}+u_{2}}{2}$, where $u_{1}$ and $u_{2}$ are single-layer potentials with surface densities $\mu_{1}$ and $\mu_{2}$, respectively, distributed over the surface of the spheres $\sigma_{R_{1}}$ and $\sigma_{R_{2}}$. However, the way, the single-layer densities $\mu_{1}$ and $\mu_{2}$ are constructed from the boundary data differs from Sect. 4.1.

We start with the representation of the potential $u_{2}$,

$u_{2}(x)=\int_{\sigma_{R_{2}}} \frac{\mu_{2}(y)}{|x-y|} \mathrm{d} \sigma_{2}(y)$.

We discretize Eq. (34) and determine the single-layer density $\mu_{2}$ at a set of points $\left\{P_{j}: j=1, \ldots, J\right\}$ as solution of the linear system

$\delta f\left(Q_{i}\right)=\sum_{j=1}^{J} \mu_{2}\left(P_{j}\right) \frac{1}{\left|P_{j}-Q_{i}\right|} w_{j}, \quad j=1, \ldots, J$, 
where $\left\{Q_{i}: i=1, \ldots, J\right\}$ are the given data points, and $\left\{w_{j}: j=1, \ldots, J\right\}$ are suitably chosen weights. Note that the solution has to be regularized so as to reduce the influence of noise in the data $\delta f$ on the solution $\mu_{2}$. We omit the details and refer to Lavrent'ev et al. (1986). We only want to remark that information about the data noise standard deviation is used to determine the regularization parameter. The latter determines how well the solution fits the data.

In order to guarantee the boundary condition, Eq. (17), we use the set of points $P_{j}$ to obtain another set of points $\tilde{P}_{j}$ by Kelvin transformation with respect to the sphere $\sigma_{R}$. With $R_{1} \cdot R_{2}=R^{2} \quad$ and $\quad R_{2}=R-h$, we obtain $R_{1}=\frac{R^{2}}{R-h} \approx R+h$. Therefore, the points $\tilde{P}_{j}$ are considered to be located on the sphere $\sigma_{R_{1}}$. We assign to the points $\tilde{P}_{j}$ the same single-layer density values as to the points $P_{j}$, i.e., we set $\left\{\mu_{1}\left(\tilde{P}_{j}\right)=\mu_{2}\left(P_{j}\right), j=1, \ldots, J\right\}$. The corresponding single-layer potential is $u_{1}$. Then, the solution to Eq. (14), $u=\frac{u_{1}+u_{2}}{2}$, still approximates the given data and its radial derivative equals exactly zero.

In this way, we have obtained a regularized solution to the highly ill-conditioned Cauchy problem, Eq. (14). Of course, alternative methods to solve the first-kind integral equation, Eq. (34), are known; however, this is beyond the scope of this paper. Moreover, in Sect. 5, we will show that the approach to be discussed in Sect. 4.3 is sufficiently accurate to meet the requirements of current geodetic practice.

\subsection{Application of the Poisson integral}

Suppose $u_{2}$ is the harmonic solution of the exterior Dirichlet problem for the sphere $\sigma_{R_{2}}$, where $R_{2}=R-h$, i.e.,

$u_{2}(x)=\frac{\left(|x|^{2}-R_{2}^{2}\right)}{4 \pi R_{2}} \int_{y \in \sigma_{R_{2}}} \frac{F(y)}{|x-y|^{3 / 2}} \mathrm{~d} \sigma_{R_{2}}(y), \quad x \in \sigma_{R_{2}, e x t}$.

We set $u_{2}(x)=\delta f(x)$ for $x \in S$, and determine the function $F$ as a solution of the first-kind integral equation, Eq. (35). We discretize the integral equation in the same way as used in Sect. 4.2. Moreover, we use the method of residuals (cf. Lavrent'ev et al. 1986), which exploits information about data noise, to find a suitable regularization parameter. Remember that the choice of the regularization parameter decides how well the solution fits the data.

The solution $u_{2}$ fulfils $\left.u_{2}\right|_{s} \approx \delta f$. Next, we define the harmonic solution of the interior Dirichlet problem for the sphere $\sigma_{R_{1}}$, where $R_{1}=R+h$,

$u_{1}(x)=\frac{\left(R_{1}^{2}-|x|^{2}\right)}{4 \pi R_{1}} \int_{y \in \sigma_{R_{1}}} \frac{F(y)}{|x-y|^{3 / 2}} \mathrm{~d} \sigma_{R_{1}}(y), \quad x \in \sigma_{R_{1}, \text { int }}$, where $F$ is given in Eq. (35). This reduces to the numerical computation of a surface integral. Finally, the solution of Eq. (14) is

$u(x)=\frac{1}{2}\left(u_{1}(x)+u_{2}(x)\right), \quad x \in \Omega$,

where $\Omega \in \mathbb{R}^{3}$ is the region enclosed by the spheres $\sigma_{R_{1}}$ and $\sigma_{R_{2}}$.

\section{Numerical results}

Three different areas have been selected to apply the methods proposed here. Geometric height anomalies are given at a set of points located inside the area. They have been obtained as differences between GPS ellipsoidal heights and normal heights. The latter have been obtained by leveling and gravimetry. When data are needed along the boundary of the area, they are obtained by thin-plate spline interpolation.

Gravimetric height anomalies are usually given on an equiangular grid. Then, they are interpolated at the location of the GPS points using also thin-plate splines. Next, differences between the interpolated gravimetric height anomalies and the given geometric height anomalies are computed. They are the input data for the computation of the function $\hat{T}$ and $\tilde{T}$, respectively. The following test areas have been selected:

1. Test area 1 is a $2^{\circ} \times 1.75^{\circ}$ area in Germany. We computed a gravimetric quasi-geoid by least-squares using radial basis functions. Geometric height anomalies at 61 points distributed over the area have been provided by Bundesamt für Kartographie und Geodäsie (BKG). They range between 0.440 and $0.690 \mathrm{~m}$. We interpolate the observed differences between gravimetric and geometric height anomalies along the boundary of the rectangular area using the program "Surface" (Smith and Wessel 1990) from the generic mapping tools (GMT) software (Wessel and Smith 2004), which is based on thin-plate splines. We apply the method of finite differences, described in Sect. 3.

2. Test area 2 is in the Netherlands. A new gravimetric quasi-geoid (GRAVGEO2006) has been computed following the approach in de Min (1996). It uses the EIGENCG03C global gravity field model (Förste et al. 2005). Differences between GPS ellipsoidal heights and levelled heights are provided at 490 points by the Adviesdienst Geo-informatie en ICT van Rijskwaterstaat (RWS-AGI), see Fig. 2. The Dutch primary height network uses levelled heights with respect to Normaal Amsterdams Peil (NAP).

For the area of the Netherlands, differences between levelled heights and normal heights are below a few 

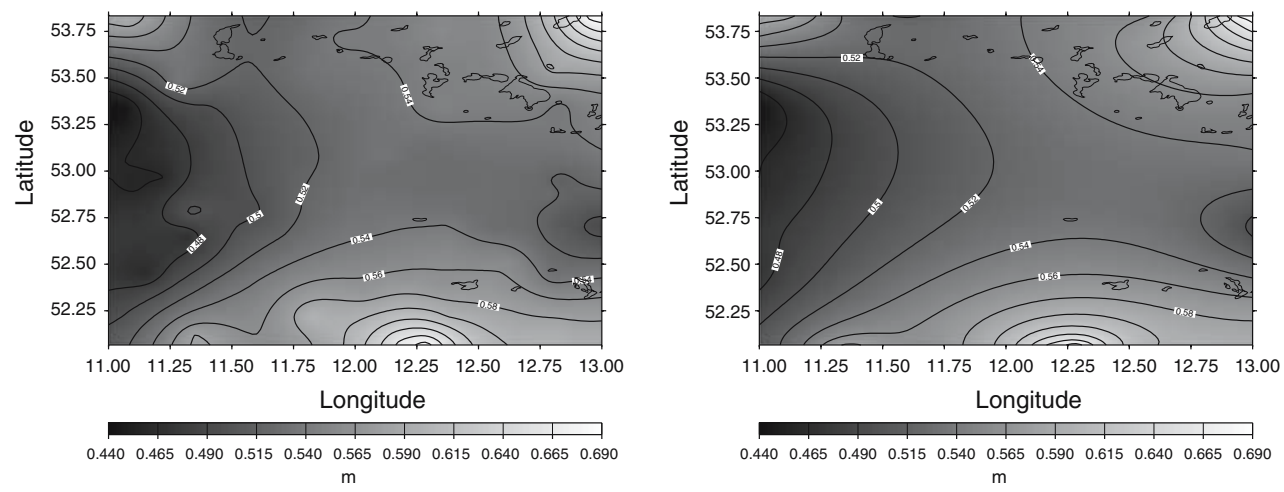

Fig. 1 Observed (left) versus predicted (right) differences between geometric and gravimetric height anomalies. The latter represent a finite difference solution of Eqs. (11) and (12). The RMS of 'observed minus predicted' is $0.7 \mathrm{~cm}$
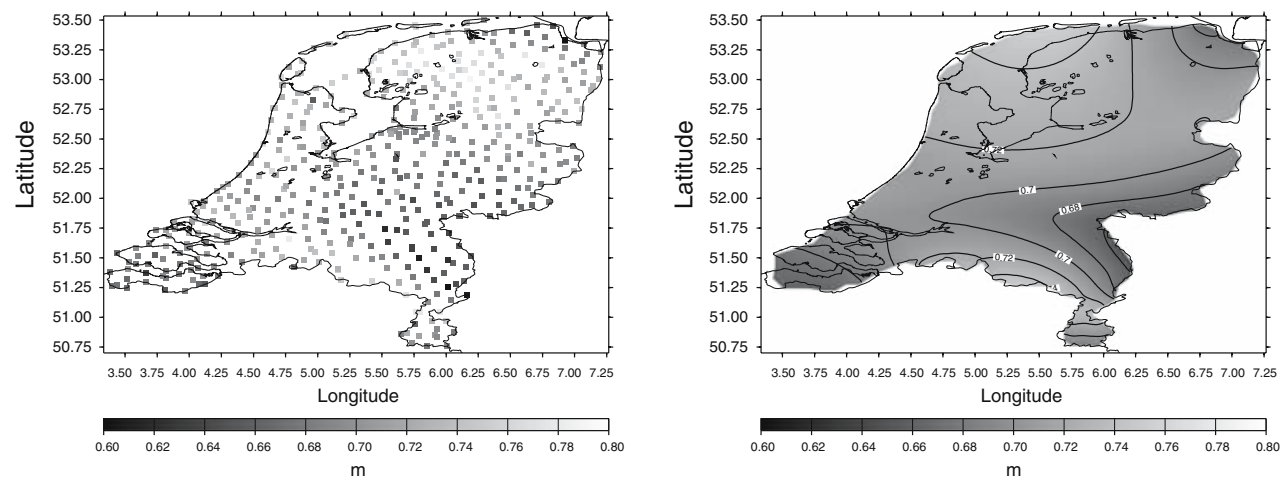

Fig. 2 Left panel: 490 data points with given differences between gravimetric and geometric height anomalies over the Netherlands. Right panel: FEM solution of Eqs. (11) and (12) using only data along the border of the Netherlands. It represents the data with an accuracy of $1.9 \mathrm{~cm}$

millimetres, due to the small horizontal gravity gradient over the country. Therefore, the levelled heights are considered as normal heights. We apply FEM and piecewise finite differences (cf. Sect. 3), and the Poisson integral method (cf. Sect. 4.3).

3. Test area 3 is in Germany. The data have been provided by the BKG. The gravimetric height anomalies are identical with EGG97 (Denker and Torge 1998). At 894 points, geometric height anomalies are given, see Fig 4. We apply the method of Sect. 4.3.

Note that, in practice, the differences between gravimetric and geometric height anomalies do not only reflect the contribution of the kernel of $D_{\Delta g}$ to the height anomalies, but may contain also systematic errors in GPS ellipsoidal heights, normal heights, and gravimetric quasi-geoid solutions. A separation between both contributors is not possible without additional information (cf. Featherstone 2004). The results presented below have to be understood in this sense.

Figure 1 shows the differences between geometric and gravimetric height anomalies inside test area 1 (left panel) and predicted differences computed from the solution $\hat{T}$ of
Eqs. (11) and (12) (right panel). The latter is obtained with the method of finite differences (cf. Sect. 3). This solution needs information about the differences along the border of the test area. Data inside the area are used to obtain these values by interpolation. They are not involved in the process of solving the problem Eqs. (11) and (12).

Nevertheless, the function $\hat{T}$ represents the data inside the area very well. The differences 'observed minus predicted' vary between -0.033 and $0.028 \mathrm{~m}$; the RMS difference is $0.007 \mathrm{~m}$. This supports the idea that observed differences between gravimetric and geometric height anomalies contain a significant contribution from the kernel of the operator $D_{\Delta g}$.

Figures 2 and 3 show the differences between geometric and gravimetric height anomalies for test area 2 (the Netherlands) and the predicted differences using different solutions: (i) a FEM solution of Eqs. (11) and (12) (cf. Fig. 2, right panel), (ii) a piecewise finite difference solution of Eqs. (11) and (12) (cf. Fig. 3, left panel), and (iii) the solution of Eq. (8) using the Poisson integral (cf. Fig. 3, right panel). Note that solutions (i) and (ii) only use the data along the boundary of the Netherlands, whereas solution (iii) uses also the data points inside the area. 
Fig. 3 Predicted differences between geometric and gravimetric height anomalies for the Netherlands. Left panel: piecewise finite difference solution of Eqs. (11) and (12). Right panel: Poisson-integral solution of Eq. (8). The latter represents the data with an accuracy of $0.3 \mathrm{~cm}$
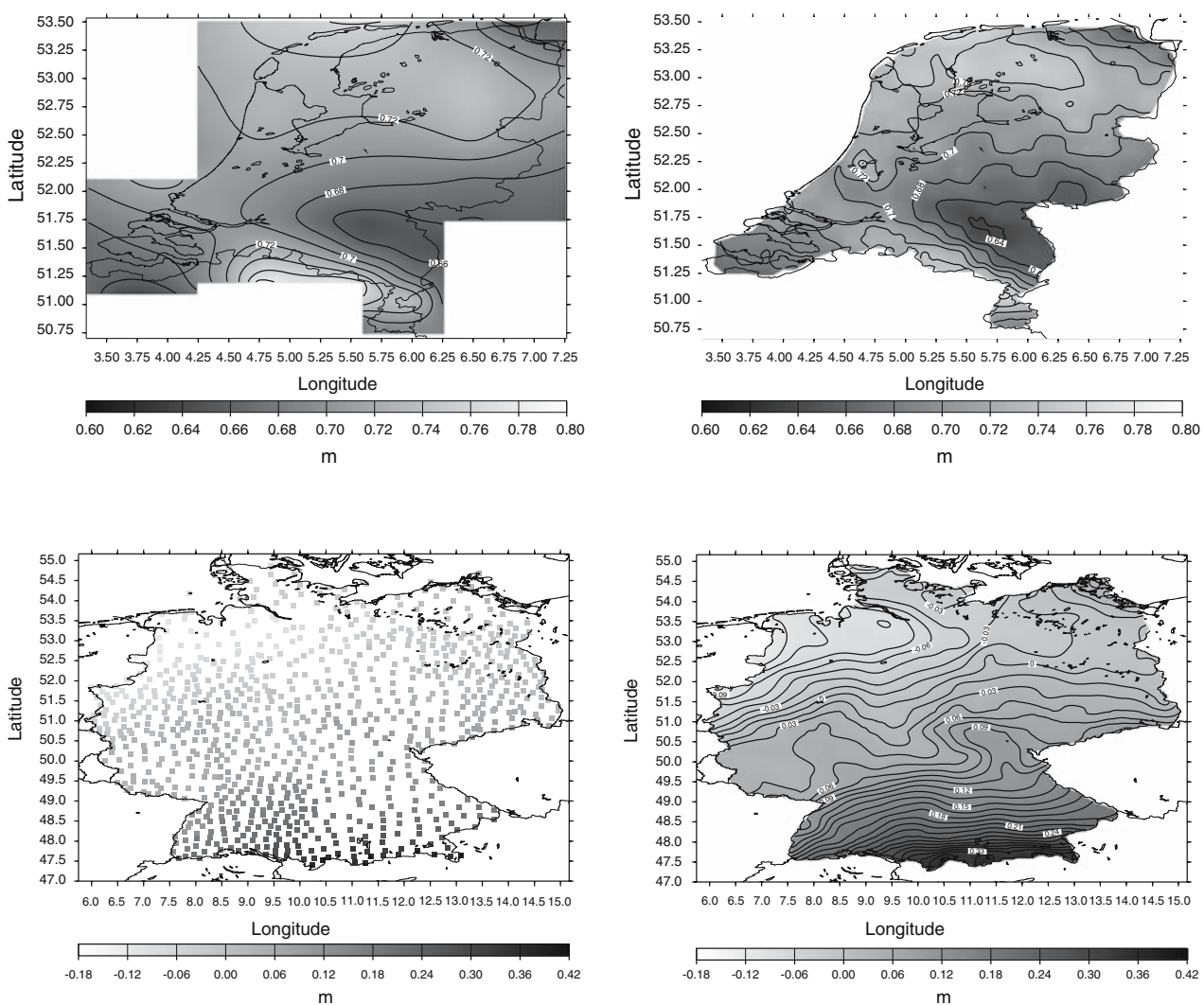

Fig. 4 Left panel: 894 data points with given differences between gravimetric and geometric height anomalies over Germany. Right panel: Poisson-integral solution of Eq. (8). The solution represents the data with an accuracy of $1 \mathrm{~cm}$
To compute the FEM solution of Eqs. (11) and (12), we use the free software package FreeFEM (Pironneau and Prud'homme, 2001). FreeFEM expects as input a triangulation of the area, which we computed using the triangulator Triangle (Shewchuk, 2002). The RMS difference, computed at 84 GPS control points distributed over the Netherlands, is $0.019 \mathrm{~m}$ (minimum $=-0.059 \mathrm{~m}$, maximum $=0.055 \mathrm{~m}$ ). Note that the solution only uses data along the border of the Netherlands.

The piecewise finite difference solution in Fig. 3, also uses the data points inside the area to interpolate data along the boundaries of the sub-rectangles. This allows us to obtain a solution that fits the data better than the FEM solution of Fig 2. The differences 'predicted minus observed' take up values between -0.027 and $0.054 \mathrm{~m}$. The RMS difference reduces from $0.019 \mathrm{~m}$ (finite element solution) to $0.015 \mathrm{~m}$.

The Poisson integral solution uses the FEM solution of Eqs. (11) and (12) as initial solution (cf. Sect. 4.3). The initial solution is improved in step 2 as outlined in Sect. 4.3. The final solution is shown in the right panel of Fig. 3. The differences 'observed minus predicted' are between -0.008 and $0.007 \mathrm{~m}$. The RMS difference is only $0.003 \mathrm{~m}$, i.e., the solution $\tilde{T}$ fits the differences between gravimetric and geometric height anomalies up to a few millimetres.

This is in agreement with our assumption about the noise standard deviation of the differences, which is used in the determination of the regularization parameter. The actual noise standard deviation for differences between gravimetric and geometric height anomalies for the Netherlands is likely to be larger. However, a proper noise modeling is beyond the scope of this study.

The two-step procedure of Sect. 4.3 is also applied to Germany. Again, a FEM solution of Eqs. (11) and (12) serves as initial solution, which is improved in the second step. BKG reported a noise standard deviation of $0.01 \mathrm{~m}$ for differences between gravimetric and geometric height anomalies, which has been used in this study. Figure 4 shows the data and the final solution. The residuals vary between -0.027 and $0.032 \mathrm{~m}$; the RMS residual is $0.007 \mathrm{~m}$. The latter is close to the a priori data noise level, which indicates that the regularization parameter has been chosen properly.

We also tested whether $\tilde{T}$ has zero radial derivatives (cf. Eq. 8). For Germany, the radial derivatives take up values between -1 and $1 \mu \mathrm{Gal}$; the RMS radial derivative is 0.4 $\mu \mathrm{Gal}$.

\section{Summary and conclusions}

We have derived a mathematical description of the non-trivial kernel of the gravity anomaly operator $D_{\Delta g}$, which arises in local gravity field modelling from gravity anomaly data. 
Functions from the kernel are harmonic and generate a zero gravity anomaly signal inside the local area. Because they are non-trivial, they contribute to the differences between gravimetric height anomalies and height anomalies derived from GPS and levelling data, which have been reported in numerous studies. To remove the non-uniqueness using geometric height anomalies provided by GPS-levelling data, a Cauchy problem for the Laplace equation has to be solved.

We have proposed several numerical procedures to compute approximate solutions of the Cauchy problem for the Laplace equation in the vicinity of the local area. When applied to real data, the solution of the Cauchy problem for the Laplace equation also absorbs systematic errors present in GPS data, levelling data and gravimetric quasi-geoid solutions that are larger than the adopted noise level in the differences between geometric and gravimetric height anomalies. Without additional information about the systematic errors, it is not possible to separate them from the contribution of non-uniqueness.

We applied the numerical procedures to different test areas, including the Netherlands and Germany. Data noise is accounted for when choosing the appropriate regularization parameter for downward continuation of the local data. For the Netherlands and Germany, we assumed a standard deviation of 0.005 and $0.01 \mathrm{~m}$, respectively. The standard deviation of the difference between the solution of the Cauchy problem and the data are close to these values, as well. This indicates that the chosen regularization procedure works correctly.

If one is only interested in an optimal transformation of GPS ellipsoidal heights into the national height system, the proposed numerical procedures provide an easy-to-handle tool to do that. The procedures are easy to implement and suited for the processing of large amount of data. Compared with parametric methods, the user does not have to make any decision about the proper choice of the model. However, the user must provide information about the noise level in the data. If the noise is underestimated, the solution will not only model signal (essentially the contribution of functions belonging to the kernel of $D_{\Delta g}$ ), but will also capture a part of the noise. Vice versa, if the noise is overestimated, the solution is too smooth and some signal is lost.

Another implication of this study is that GPS-levelling data should not be used to validate local quasi-geoid models obtained from terrestrial gravity anomalies. They carry information, which is not contained in a gravimetrically computed quasi-geoid. Hence, they are mandatory to obtain a unique local quasi-geoid.

A "corrector surface" is often used in local quasi-geoid modelling to account for the various systematic errors in geometric and gravimetric data. From the non-uniqueness point of view of the local quasi-geoid modelling from gravity anomalies, such a "corrector surface" could be used as a first-order correction for the non-uniqueness, which needs further refinements. This is also the idea behind the LSC approach discussed in Kotsakes and Sideris (1999). However, all these approaches do not take the mathematical nature of the non-uniqueness into account, hence may not provide optimal solutions.

Acknowledgment The Bundesamt für Kartographie und Geodäsie (BKG) provided geometric height anomalies over Germany. Kadaster and Dienst Geoinformatie en ICT of Rijkswaterstaat provided geometric height anomalies over the Netherlands. This support is gratefully acknowledged. We also acknowledge the detailed comments of three anonymous reviewers, which helped us to improve the manuscript.

\section{References}

de Bruijne AJT, Haagmans RHN, de Min EJ (1997) A preliminary North Sea Geoid model GEONZ97. Rep MDGAP-9735, Directoraat-Generaal Rijkswaterstaat, Meetkundige Dienst, Delft

Denker H, Torge W (1998) The European Gravimetric Quasigeoid EGG97-An IAG Supported Continental Enterprise. In: Forsberg R, Feissel M, Dietrich R (ed) Geodesy on the move-gravity, geoid, geodynamics and antarctica, IAG Symp vol 119. Springer, Heidelberg pp 249-254

Dinter G, Illner M, Jäger R (1997) A synergetic aproach for the integration of GPS heights into standard height systems and for the quality control and refinement of geoid models. Proc Subcommission for Europe Symposium EUREF, Ankara 1996. In: Bayerische Akademie der Wissenschaften (ed) Veröffentlichungen der Internationalen Erdmessung der Bayerischen Akademie der Wissenschaften, vol 57, Beck'sche Verlagsbuchhandlung, München

Ebenfelt P, Shapiro HS (1995) The Cauchy-Kowalevskaya theorem and generalizations. Commun Partial Diff Equ 20:939-960

Featherstone WE (1998) Do we need a gravimetric geoid or a model of the base of the Australian Height Datum to transform GPS heights? Aust Surv 43:273-280

Featherstone WE (2000) Refinement of a gravimetric geoid using GPS and levelling data. J Surv Eng 126:27-56

Featherstone WE (2004) Evidence of a north-south trend between AUSGeoid98 and the AHD in southwest Australia. Surv Rev 37: 334-343

Förste C, Flechtner F, Schmidt R, Meyer U, Stubenvoll R, Barthelmes F, König R, Neumayer KH, Rothacher M, Reigber Ch, Biancale R, Bruinsma S, Lemoine J-M, Raimondo JC (2005) A new high resolution global gravity field model derived from combination of GRACE and CHAMP mission and altimetry/gravimetry surface gravity data. Paper presented at EGU General Assembly 2005, Vienna, April. Available as http://www.gfz-potsdam. de/pb1/op/grace/results/grav/g004_EGU05-A-04561.pdf

Fotopoulos G, Kotsakis C, Sideris MG (2003) How accurately can we determine orthometric height differences from GPS and geoid data. J Surv Eng 1:1-10

Fotopoulos G (2005) Calibration of geoid error models via a combined adjustment of ellipsoidal, orthometric and gravimetric geoid height data. J Geod 79:111-123

Grebenitcharsky RS, Rangelova EV, Sideris MG (2005) Transformation between gravimetric and GPS/levelling-derived geoids using additional gravity information. J Geodyn 39:527-544

Hadamard J (1921) Lectures on Cauchy's problem in linear partial differential equations. Silliman Lectures Series, Yale University Press, New Haven

Heiskanen WA, Moritz H (1967) Physical geodesy. Freeman, San Francisco 
Ito K (ed) (1980) Harmonic functions and subharmonic functions: invariance of harmonicity. Encyclopedic dictionary of mathematics, 2nd edn. MIT Press, Cambridge, p 725

Jäger R (1999) State of the art and present developments of a general concept for GPS-based height determination. In: Lilje M (ed) Proc of the symposium geodesy and surveying in the future-the importance of heights, gävle, Sweden, March, Report in Geodesy and Geographical Information Systems, LMV-Rapport 1999(3):161174

Jäger R (2000) State of the art and present developments of a general concept for GPS-based height determination. In: Proc 1st workshop on GPS and mathematical geodesy in Tanzania, UCLAS, Dar Es Salaam, October 1999

Jäger R, Schneid S (2001) Online and postprocessed GPS-heighting based on the concept of a digital height reference surface (DFHRS). In: Drewes H, Dodson AH, Fortes LPS, Sanchez L, Sandoval P (eds) Vertical reference systems, Cartagena, Colombia, Feb 2001, IAG Symp vol 124. Springer, Heidelberg, pp 203-208

Jost J (2002) Partial differential equations. Springer, New York

Kotsakis C, Sideris MG (1999) On the adjustment of combined GPS/ levelling/geoid networks. J Geod 73:412-421

Lavrent'ev MM, Romanov VG, Shishatskii SP (1986) Ill-posed problems of mathematical physics and analysis. American Mathematics Society, Providence

Lysaker DI, Omang OCD, Pettersen BR, Solheim D (2007) Quasigeoid evaluation with improved levelled height data for Norway. J Geod. DOI: $10.1007 / \mathrm{s} 00190-006-0129-6$ de Min E (1996) De geoide voor Nederland. Nederlandse Commissie voor Geodesie, Groene serie, vol 34, Delft

Nahavandchi N, Soltanpour A (2006) Improved determination of heights using a conversion surface by combining gravimetric quasi-geoid/geoid and GPS-levelling height differences. Stud Geophys Geod 50:165-180

Pironneau O, Prud'homme C (2001) FreeFEM user manual. http://www. freefem.org

Sacerdote F, Sansò F (1987) New developments of boundary value problems in physical geodesy. In Proc IAG symp, Vancouver, vol 2, pp 369-390

Shewchuk JR (2002) Delaunay refinement algorithms for triangular mesh generation. Comput Geom Theory Appl 22(1-3):21-74

Sjöberg LE (2005) A discussion on the approximations made in the practical implementation of the remove-compute-restore technique in regional geoid modelling. J Geod 78:645-653

Soltanpour A, Nahavandchi H, Featherstone WEE (2006) The use of second-generation wavelets to combine a gravimetric quasigeoid model with GPS-levelling data. J Geod 80(3):82-93, DOI: 10.1007/s00190-006-0033-0

Smith WHF, Wessel P (1990) Gridding with continous curvature splines in tension. Geophys 55:293-305

Vanicek P, Castle RO, Balazc EI (1980) Geodetic leveling and its applications. Rev Geophys 18:505-524

Wessel P, Smith WHF (2004) The Generic Mapping Tools (GMT) Technical Reference and Cookbook, Version 4.0. SOEST/NOAA, Univ Hawai'i, Manoa 